\title{
Effect of Two Different Hydrating Solutions on Antibacterial Property of White Mineral Trioxide Aggregate Against Enterococcus Faecalis - An in Vitrocomparative Study
}

\author{
Dr. Venkatesh Babu NS ${ }^{1}$, Dr Ganga. A. ${ }^{2}$, Dr. Parin V. Bhanushali ${ }^{3}$ \\ ${ }^{I}$ (Professor And Head, Department Of Pediatric And Preventive Dentistry, V. S. Dental College And Hospital, \\ Bangalore, India) \\ ${ }^{2,3}$ (Post Graduate Student, Department Of Pediatric And Preventive Dentistry, V. S. Dental College And \\ Hospital, Bangalore, India)
}

\begin{abstract}
:
Background: Antimicrobial activity of root canal materials may help to eliminate residual microorganisms unaffected by the effects of both chemo-mechanical preparation and intracanal medication. Mineral trioxide aggregate (MTA), is one such bioactive material that has potential antimicrobial property. The principle aim of this study was to evaluate the antibacterial properties of ProRoot WMTA against E. faecalis and to compare the antibacterial effect of WMTA when mixed with 2\% Chlorhexidine (CHX), and Phosphate Buffered Saline (PBS), as a substitute for sterile water against Enterococcus faecalis (E. faecalis).

Method: ProRoot WMTA was divided into three groups one for each hydrating agent. The antimicrobial effect was determined by placing freshly mixed MTA samples in wells created in trypticase soy agar (TSA) plates inoculated with Enterococcus faecalis. These plates were maintained at room temperature for 2 hours for prediffusion of the materials and incubated at $37^{\circ} \mathrm{C}$ for 24 hours and zones of inhibitions were recorded.

Results: The samples of ProRoot WMTA irrespective of the mixing agent showed antimicrobial activity against E. faecalis. Higher mean zone of inhibition was shown by WMTA/ CHX followed by WMTA/PBS and WMTA/Sterile Water. The difference between the three groups were found to be statistically significant with $P$ value $<0.001$.

Conclusion: Phosphate buffered saline (PBS) and $2 \%$ Chlorhexidine enhanced the antibacterial efficacy to inhibit E. faecalis and can be recommended to be used as mixing agent for WMTA as substitute to sterile water provided by manufacturer.
\end{abstract}

Keywords:ProRoot WMTA, 2\% Chlorhexidine, Phosphate Buffered Saline, Enterococcus faecalis

\section{Introduction}

Microorganisms play a pivotal role in the initiation and progression of dental caries and periapical pathologies in both primary and permanent teeth. Enterococcus faecalis is a facultative anaerobic Gram-positive coccus, that is commonly detected in asymptomatic, persistent endodontic infections and is considered as one of the most resistant microorganisms to calcium hydroxide-based intracanal medications. ${ }^{1}$ Inclusion of $2 \%$ chlorhexidine (CHX) in combination with sodium hypochlorite are currently the most effective methods to combat E. faecalis. Chlorhexidine is being incorporated in root canal materials for improving prognosis by enhancing the antimicrobial properties of endodontic materials. The use of filling materials with an antimicrobial property represents one of important aspects for achieving success in endodontic therapy. Root canal filling materials should be able to eliminate residual pathogens, neutralize their toxic products and prevent canal reinfection to create a favourable environment for the healing process to proceed. It is therefore important that root canal filling materials used in primary teeth and permanent have antimicrobial activity. ${ }^{2,3}$

Mineral trioxide aggregate (MTA), is one such bioactive material with antimicrobial property that was pioneered by Dr. Torabinejad of the University of Loma Linda in California, USA in the early 1990s. ${ }^{4}$

MTA in the practice of paediatric dentistry has diverse applications. It is used as an apical barrier in immature non-vital teeth and in the coronal fragment of fractured roots, as a pulpotomy medicament in primary and permanent teeth, a pulp capping agent in young permanent teeth, and as a repair material for perforation and resorptive defects. ${ }^{5}$

One of the characteristics of MTA is its ability to form an apatite-like layer on its surface when it comes in contact with physiological fluids in vivo or with simulated body fluids such as phosphate buffer saline (PBS) ${ }^{6,7}$ Similar precipitates are also formed at the MTA-dentin interface which helps in the achievement of a good marginal seal thereby enhancing its antimicrobial activity by preventing microleakage.

Thus, the purpose of this in vitro study was to evaluate and compare the antibacterial activity of ProRoot White Mineral trioxide aggregate (WMTA) mixed with sterile (MTA) water provided by the 
manufacturer and two different hydrating solutions, i.e. 2\% Chlorhexidine and phosphate buffered saline as a substitute to MTA water against Enterococcus faecalis using the agar diffusion test (ADT).

\section{Materials And Methods}

The present comparative in vitro study was conducted in the Department of Pediatric \& Preventive Dentistry, V. S. Dental College \& Hospital, Bangalore, India and the microbiological analysis was carried out in the Department of Microbiology, Kempegowda Institute of Medical Sciences, Bangalore, India.

Samples were divided into three experimental groups.

Group 1:WMTA / Sterile water

Group 2:WMTA / 2\% chlorhexidine

Group 3:WMTA / Phosphate buffered saline

The test microorganism E. faecalis was first rehydrated with trypticase soy broth (TSB) to check viability and subcultured to trypticase soy agar (TSA) with 5\% sheep blood. The resultant agar and sheep blood mixture plate was incubated at $37^{\circ} \mathrm{C}$ overnight. Colonies were harvested from the TSA and suspended in TSB. Turbidity was adjusted by adding E. faecalis suspended in TSB to equal a $0.5 \mathrm{McFarland}$ turbidity standard. A sterile cotton swab was used to inoculate the bacterial suspension on another plate named TSA-I plate for lawn growth. Agar plates were prepared for diffusion test after plating 100 microlitre aliquots of E.fecalis. Three wells of 5-mm diameter were punched at equal distance in 4 separate TSA- I plates. ${ }^{8}$

White ProRoot-MTA (DENTSPLY Tulsa dental) was then mixed according to the manufacturer's instructions using sterile spatula and sterile glass slab by mixing $200 \mathrm{mg}$ powder with $0.72 \mathrm{ml}$ sterile water. Each group was mixed exactly the same way by substituting the test liquid i.e. $2 \%$ Chlorhexidine gluconate (Deor Dental and oral care) and freshly prepared Phosphate Buffered Saline. The resultant mixture was divided into four equal parts and transferred using a new sterile amalgam carrier and gently condensed into place to a $5 \mathrm{~mm}$ well created on each of the TSA-I plate so that each of the four experimental agar plates had one well filled with the WMTA/ sterile water mixture, WMTA/ PBS mixture and WMTA/ CHX mixture. (Fig-1) The plates were maintained at room temperature for 2 hours for pre-diffusion of the materials. Then the TSA-I plates were incubated at $37^{\circ} \mathrm{c}$ for 24 hours and zone of inhibition were measured to nearest $\mathrm{mm}$ using zone measuring scale and recorded. (Fig-2)

\section{Results}

The mean zone of inhibition was calculated for each of the three groups and compared with each other at 24- hour interval using Kruskal-Wallis test and the p-value for level of significance was calculated.

- Group -I (WMTA mixed with sterile water) showed $6 \mathrm{~mm}$ minimum and $7 \mathrm{~mm}$ maximum of zone of inhibition against $\mathrm{E}$. faecalis at 24 hours interval.

- Group -II (WMTA mixed with chlorhexidine) showed $9 \mathrm{~mm}$ minimum and $10 \mathrm{~mm}$ maximum of zone of inhibition against E. faecalis at 24 hours interval.

- Group-III (WMTA mixed with phosphate buffered saline) Showed $6 \mathrm{~mm}$ minimum and $7 \mathrm{~mm}$ maximum zone of inhibition against E. faecalis at 24 hours interval.

Higher mean zone of inhibition of $9.17 \mathrm{~mm}$ was recorded in Group-II (WMTA/ CHX) followed by Group-III (WMTA/PBS) with $6.83 \mathrm{~mm}$ and Group-I (WMTA/STERILE WATER) recorded $6.17 \mathrm{~mm}$. The difference in mean zone of inhibition amongst each of the three groups was calculated and compared for statistical significance using Mann-Whitney test and it was found to be statistically significant between all the groups. (TABLE-1, GRAPH -1)

Difference in mean zone of inhibition between WMTA mixed with Sterile water (Group-I) \&WMTA mixed with $2 \%$ chlorhexidine (Group-II) was calculated as 3.0 and the p-value was <0.01 (TABLE-2, GRAPH 1).Difference in mean zone of inhibition between WMTA mixed with Sterile water (Group I) \&WMTA mixed with Phosphate buffered saline (Group III) was calculated as 0.667 and the p-value was <0.05 (TABLE-2, GRAPH -1), and

Difference in mean zone of inhibition between WMTA mixed with $2 \%$ chlorhexidine (Group-II) \&WMTA mixed with Phosphate buffered saline (Group-III) was calculated as 2.33 the p-value was $<0.01$ (TABLE-2, GRAPH -1). 
Effect of two different hydrating solutions on antibacterial property of white mineral ....

Table-1:Mean zones of inhibition and Kruskal-Wallis analysis

\begin{tabular}{|l|l|l|l|l|l|l|l|}
\hline Group & $\begin{array}{l}\text { Mean } \\
\text { mm }\end{array}$ & $\begin{array}{l}\text { Std. } \\
\text { dev }\end{array}$ & $\begin{array}{l}\text { SE of } \\
\text { Mean }\end{array}$ & Min & Max & $\begin{array}{l}\text { Kruskal-Wallis } \\
\text { Chi-square }\end{array}$ & P-Value \\
\hline Group I & 6.17 & 0.41 & 0.17 & 6 & 7 & \multirow{2}{*}{} & \multirow{2}{*}{.001* } \\
\cline { 1 - 6 } Group II & $\mathbf{9 . 1 7}$ & $\mathbf{0 . 4 1}$ & $\mathbf{0 . 1 7}$ & $\mathbf{9}$ & $\mathbf{1 0}$ & \\
\hline Group III & $\mathbf{6 . 8 3}$ & $\mathbf{0 . 4 1}$ & $\mathbf{0 . 1 7}$ & $\mathbf{6}$ & $\mathbf{7}$ & & \\
\hline
\end{tabular}

*denotes significant difference

Table-2: Intergroup comparison

\begin{tabular}{|c|c|c|c|c|c|c|}
\hline \multirow{2}{*}{ (I) Group } & \multirow{2}{*}{ (J) Group } & \multirow{2}{*}{$\begin{array}{l}\text { Mean Difference } \\
\text { (I-J) }\end{array}$} & \multirow{2}{*}{$\begin{array}{l}\text { Std. } \\
\text { Error }\end{array}$} & \multirow{2}{*}{ P-Value } & \multicolumn{2}{|c|}{$\begin{array}{llll}95 \% & \text { Cl } & \text { for } & \text { mean } \\
\text { difference }\end{array}$} \\
\hline & & & & & $\begin{array}{l}\text { Lower } \\
\text { Bound }\end{array}$ & $\begin{array}{l}\text { Upper } \\
\text { Bound }\end{array}$ \\
\hline \multirow{2}{*}{ Group I } & Group II & -3.000 & 0.236 & 0.002* & -3.635 & -2.365 \\
\hline & Group III & -0.667 & 0.236 & 0.027* & -1.302 & -0.032 \\
\hline \multirow{2}{*}{ Group II } & Group I & 3.000 & 0.236 & $0.002 *$ & 2.365 & 3.635 \\
\hline & Group III & 2.333 & 0.236 & $0.002 *$ & 1.698 & 2.968 \\
\hline \multirow{2}{*}{ Group III } & Group I & 0.667 & 0.236 & $0.027 *$ & 0.032 & 1.302 \\
\hline & Group II & -2.333 & 0.236 & $0.002 *$ & -2.968 & -1.698 \\
\hline
\end{tabular}

*denotes significant difference

Graph-1: Mean Zone of Inhibition (mm) in the groups
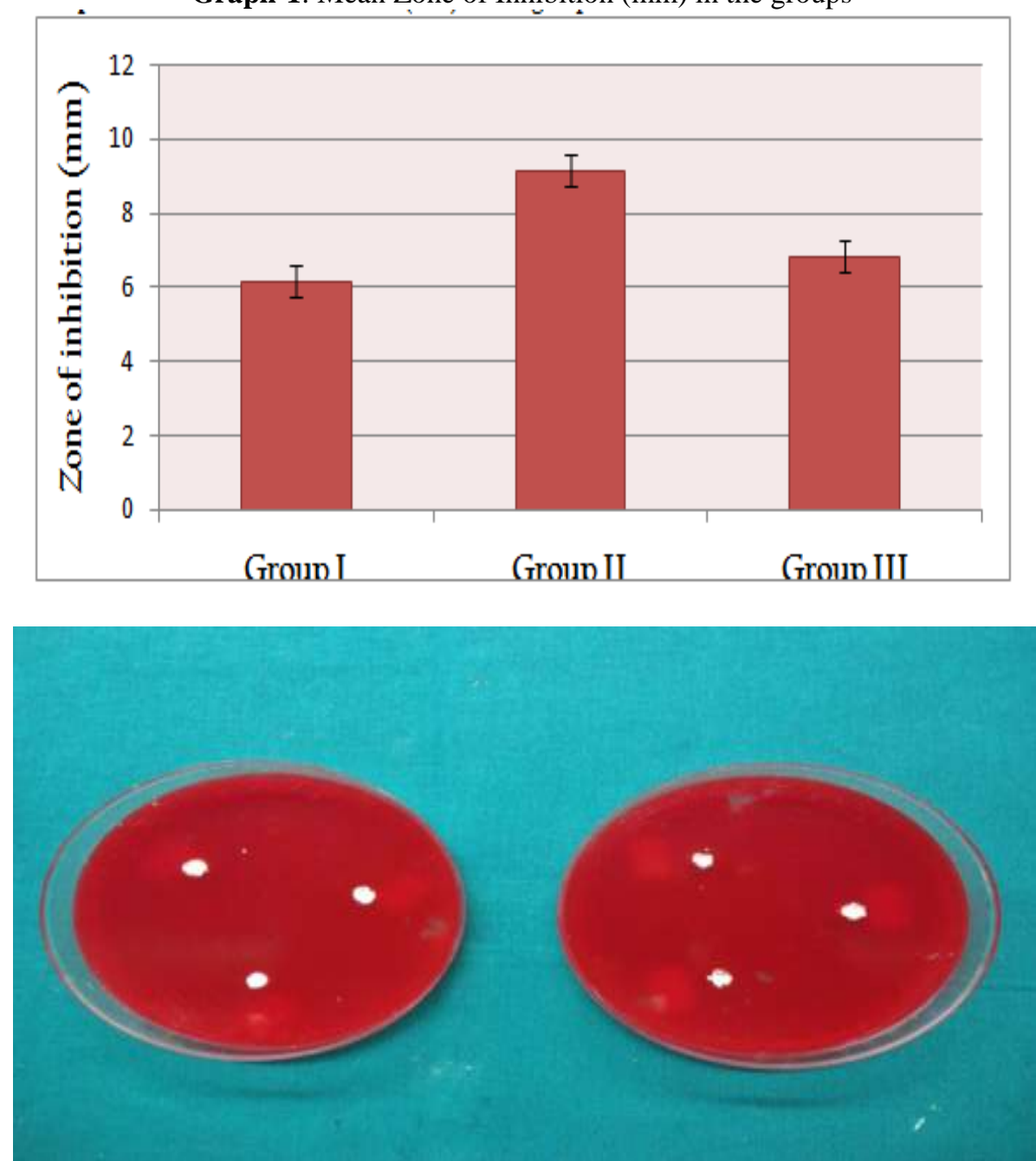

Figure-1: Placement of WMTA into wells after mixing with hydrating agents 


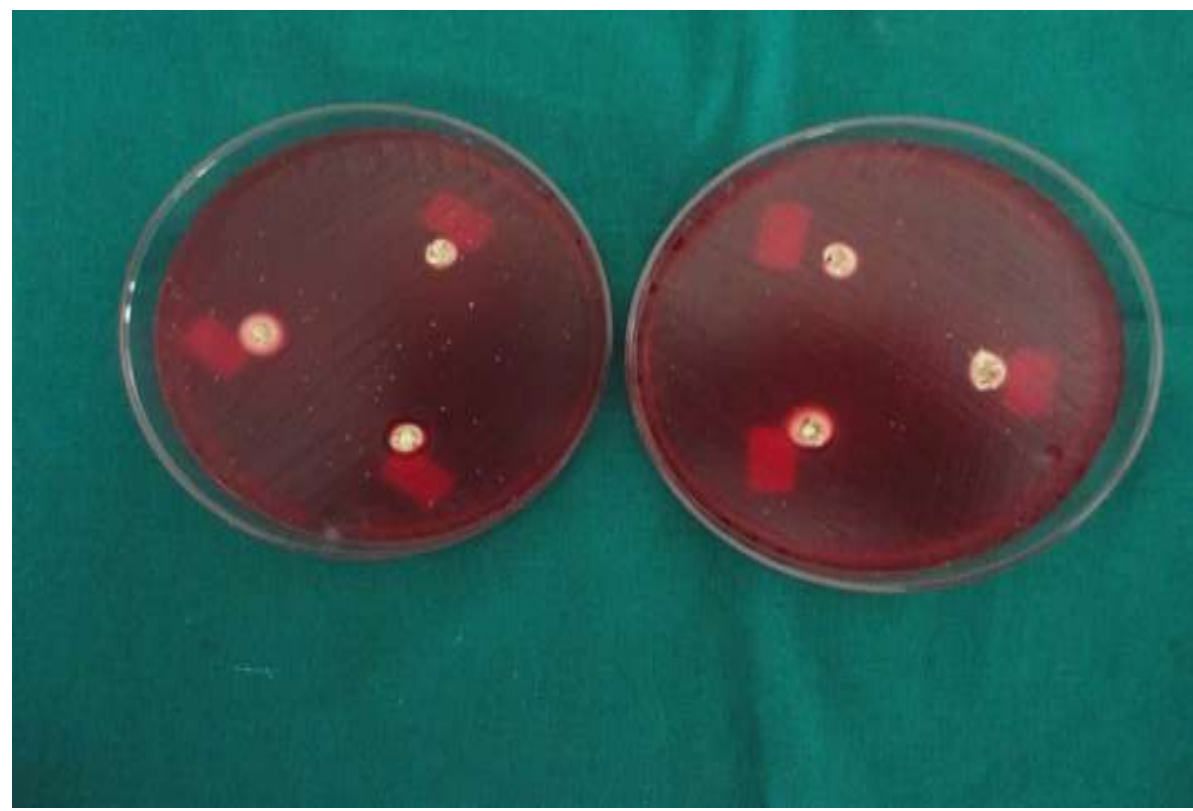

Figure-2:Zones of inhibition after 24hr incubation

\section{Discussion}

The present study was conducted to investigate the antimicrobial activity of WMTA against E. faecalis, a very resistant microorganism that is present in root canals of primary and permanent teeth with necrotic pulp and apical periodontitis and is commonly found in teeth that need endodontic retreatment. The antibacterial effect of WMTA mixed with $2 \%$ chlorhexidine and PBS was also assessed and compared. An intracanal dressing of $2 \%$ chlorhexidine has shown to eradicate E. faecalis from dentinal tubules and the root canal space. MTA stored in PBS helps in biomineralization improving its sealing ability by preventing bacterial leakage. As per the published data available, there are no reports of studies conducted to evaluate PBS as a mixing agent for MTA. Hence in the present study, an attempt was made to use PBS as hydrating agent for WMTA and test its antimicrobial efficacy.

The results of the present study show that ProRoot WMTA with water is capable of inhibiting E. feacalis and are comparable with the studies conducted by Asgary et al $(2007,2008)^{9,10}$ FabianePivaa $(2008)^{11}$, Maha A. Mahmood (2010) ${ }^{12}$ using agar diffusion test against various species of microorganisms in-vitro and the diameters of zones of inhibition were found around MTA against E. faecalis. The mean zone of inhibition in the present study for WMTA mixed with water was $6.17 \mathrm{~mm}$. Ayce Unverdi Eldeniz ${ }^{13}$ used direct contact test (DCT) with Staphylococcus aureus, Enterococcus faecalis, and Pseudomonas aeruginosa, and reported that ProRoot MTA in their study delayed or limited growth of E. faecalis.

In contrast to the results of the present study, Estrela et al $(2000)^{14}$, Yoshiyuki Yasuda et al (2008) ${ }^{15}$, Mohammad Hasan Zarrabi (2009) ${ }^{16}$ who investigated the antibacterial effect of some root end filling materials, observed that MTA had no antibacterial activity against E. faecalis.

The antimicrobial effect of MTA is attributable to its high $\mathrm{pH}$ or release of diffusible calcium hydroxide into the growth medium. It has been reported that $\mathrm{E}$. faecalis shows resistance to intra-canal dressings containing calcium hydroxide. ${ }^{13}$ Daniela Cristina Miyagak $(2006)^{17}$ observed that the microorganism E. faecalis was resistant against all sealers tested in their study. Even though $\mathrm{pH}$ reached 12.5 , antimicrobial activity in Portland cement and MTA was not observed.

According to Stowe et al. $(2004)^{8}$ the results of the studies vary due to several reasons, one of them could be method of placement. Another explanation is that different formulations of MTA which are used. Khalid Al-Hezaimi (2009) ${ }^{18}$, who assessed the antimicrobial effects of 4 mineral trioxide aggregate (MTA) preparations against C. albicans and E. faecalis in vitro found that the origin of MTA as well as the type of preparation might affect its antimicrobial characteristics.

We have used TSA with 5\% sheep blood as culture medium. The agar diffusion method, is the most commonly employed technique for evaluation of antimicrobial activity. The differences in agar medium, diffusion capacity of inhibitory agents, bacterial strains and cellular density may interfere with the formation of inhibition zones around materials used in antimicrobial testing. ${ }^{13}$

The effect of CHX to inhibit E. faecalis has been reported previously. Stowe et al (2004) who were the first to use MTA with CHX to check the hypothesis that the antimicrobial properties of ProRoot MTA would improve with the addition of $0.12 \%$ chlorhexidine, showed significantly larger zones of inhibition 
$(13.5 \mathrm{~mm}){ }^{8}$ The inhibition zone of WMTA/CHX $(9.17 \mathrm{~mm})$ in the current study is comparable with the study conducted by Stowe et al.

Dennis M. Holt (2007) ${ }^{19}$ reported the zones of inhibition were significantly larger for samples mixed with $2 \%$ chlorhexidine but MTA mixed with sterile water always had higher compressive strengths than MTA mixed with chlorhexidine. Similarly, in the present study, zones of inhibition were larger with WMTA mixed with $2 \%$ CHX compared to other two groups. However, the mechanical properties such as the compressive strength were not tested.

Mahmut Sumer (2006) ${ }^{20}$ in their histopathological study have reported that MTA mixed with CHX materials were surrounded by fibrous connective tissue, indicating that they were well tolerated by the tissues, therefore, MTA/CHX seemed to be biocompatible when examined. Thus, in the present study, CHX was incorporated to enhance the antimicrobial effect of WMTA. The results showed that WMTA mixed with CHX indeed enhances the antibacterial efficacy against $\mathrm{E}$. faecalis.

The antimicrobial efficacy of MTA against E. faecalis when mixed with PBS as a hydrating agent was also assessed. The hydration of MTA surface and the release of calcium from MTA on contact with phosphorous of PBS produces hydroxyapatite crystals over MTA that influences the bioactivity and sealing ability. ${ }^{21,22}$ Masoud Parirokh (2009) ${ }^{21}$ determined the bacterial leakage of MTA used as a root-end filling material against Enterococcus faecalis and noted that the teeth placed in PBS showed significantly less bacterial penetration in comparison with the roots that were stored in normal saline $(\mathrm{P}<0.05)$. Considering the above factors, an attempt was made to use PBS as a hydrating agent and check if it enhances the antimicrobial efficacy of MTA. MTA mixed with PBS showed mean zone of inhibition of $6.83 \mathrm{~mm}$ that was greater than MTA mixed with sterile water samples $(6.17 \mathrm{~mm}, \mathrm{p}-<0.05)$ but lesser than the MTA/CHX group $(9.17 \mathrm{~mm}, \mathrm{p}<0.01)$.

\section{Conclusion}

Within the limits of the present study it can be concluded that ProRoot White MTA (WMTA) showed significant antibacterial activity against Enterococcus faecalis. Irrespective of the mixing agent, Enterococcus faecalis was inhibited by WMTA. 2\% Chlorhexidine solution mixed with ProRoot WMTA showed highest zone of inhibition against Enterococcus faecalis followed by ProRoot WMTA mixed with Phosphate buffered saline (PBS) and sterile water respectively.

Thus, 2\% Chlorhexidine and Phosphate buffered saline can be recommended to be used as mixing agent for WMTA. However, further studies should be conducted for the effects on mechanical properties when CHX and PBS are used as hydrating agents.

\section{References}

[1]. Onçag O, Cogulu D, Uzel A. Efficacy of various intracanal medicaments against Enterococcus faecalis in primary teeth: an in vivo study. J ClinPediatr Dent 2006;30:233-237.

[2]. Leonardo MR, Silva LAB, TanomaruFilho M, Bonifácio KC, Ito IY. In vitro evaluation of antimicrobial activity of sealers and pastes used in endodontics. J Endod 2000;26:391-394.

[3]. Sipert CR, Hussne RP, Nishiyama CK, Torres SA. In vitro antimicrobial activity of Fill Canal, Sealapex, Mineral Trioxide Aggregate, Portland cement and EndoREZ.IntEndod J 2005;38:539-543.

[4]. Camilleri J. The chemical composition of mineral trioxide aggregate.J Conserv Dent 2008;11:141-3.

[5]. VidyaSrinivasan, Paula Waterhouse \& John Whitworth. Mineral Trioxide Aggregate In Paediatric Dentistry. International Journal of Paediatric Dentistry 2009; 19: 34-47.

[6]. Hench LL, Wilson. J. Surface-active biomaterials. Science 1984; 226: 630-636.

[7]. Ducheyne P, El-Ghannam A, Shapiro. Effect of bioactive glass template on osteoblast proliferation and in vitro synthesis of bonelike tissue. J Cell Biochem1994 ;56: 162-167.

[8]. Stowe TJ, Sedgley CM, Stowe B, Fenno JC. The effects of chlorhexidinegluconate $(0.12 \%)$ on the antimicrobial properties of toothcoloredProRoot mineral trioxide aggregate. J. Endod 2004; 30(6):429-31.

[9]. AsgarySaeid,AkbariKamraniF,Taheri S. Evaluation Of Antimicrobial Effect Of MTA, Calcium Hydroxide, And Cem Cement. Iranian Endodontic Journal 2007; 2(3):105-109.

[10]. SaeedAsgary and FarshidAkbariKamrani.Antibacterial effects of five different root canal sealing materials.J. Oral Sci 2008; 50: 469-474.

[11]. FabianePivaa, Italo Medeiros FaracoJuniorb, Carlos Estrelac Antimicrobial Activity of Different Root Canal Filling Pastes Used in Deciduous Teeth.Materials Research 2008;11(2):171-173.

[12]. Maha A. Mahmood. Determination of the antibacterial activity of MTA, and pulpotec filling materials against selected microorganisms, in vitro study. J BaghColl Dentistry 2010;22(4):115-118.

[13]. AyceUnverdiEldeniz ,HasanHuseyinHadimli , HanifeAtaoglu, Dag Ørstavik . Antibacterial Effect of Selected Root-End Filling Materials. J Endod 2006; 32(4): 345-349.

[14]. Carlos Estrela, LiliLuschkeBammann, Cyntia Rodrigues A. Estrela ,Reginaldo Santana Silva, Jesus DjalmaPécora. Antimicrobial and Chemical Study of MTA, Portland Cement, Calcium Hydroxide Paste, Sealapex and Dycal. Braz Dent J 2000; 11(1): 3-9

[15]. Yoshiyuki Yasuda, ArihideKamaguchi, Takashi Saito. In vitro evaluation of the antimicrobial activity of a new resin-based endodontic sealer against endodontic pathogens.J. Oral Sci 2008;50:309-313.

[16]. Mohammad Hasan Zarrabi, Maryam Javidi, MahboubeNaderinasab and Maryam. Comparative evaluation of antimicrobial activity of three cements: new endodontic cement (NEC), mineral trioxide aggregate (MTA) and Portland Gharechahi. J Oral Sci 2009;51: 437-442. 
[17]. Daniela Cristina Miyagak, Elaine Manso Oliveira Franco de Carvalho, Carlos Roberto Colombo Robazza, Jorge KleberChavasco,GustavoLabegallineLevorato. In vitro evaluation of the antimicrobial activity of endodontic sealers.Braz Oral Res 2006;20(4):303-6.

[18]. Khalid Al-Hezaimi, Thakib A. Al-Shalan, JafarNaghshbandi, James H.S. Simon, IlanRotstein. MTA preparations from different origins may vary in their antimicrobial activity. OOOE 2009; 107(5): e85-e88.

[19]. Dennis M. Holt, J. Dustin Watts, Thomas J. Beeson, Timothy C. Kirkpatrick, Richard E. Rutledge. The Anti-microbial Effect Against Enterococcus faecalis and the Compressive Strength of Two Types of Mineral Trioxide Aggregate Mixed With Sterile Water or 2\% Chlorhexidine Liquid. J Endod 2007; 33(7): 844-847

[20]. Mahmut Sumer, MehtapMuglali, EmreBodrumlu,TolgaGuvenc. Reactions of Connective Tissue to Amalgam, Intermediate Restorative Material, Mineral Trioxide Aggregate, and Mineral Trioxide Aggregate Mixed With Chlorhexidine. J Endod 2006; 32( 11 ): 1094-1096

[21]. Parirokh M, AsgarySaeid, Eghbal M.J., GhodousiJamileh,Brink F.,Askari Far S.,Torabinezhad M.,Raouf M. The Long-Term Effect Of Saline And Phosphate Buffer Solution On Mta: An Sem And Epma Investigation. Iranian Endodontic Journal 2007; 2(3):81-86.

[22]. Linlin Han, Takashi Okiji, SeigoOkawa. Morphological and chemical analysis of different precipitates on mineral trioxide aggregate immersed in different fluids.Dental Materials Journal 2010;29(5):512-517. 